\title{
The Unique Positive Solution for Singular Hadamard Fractional Boundary Value Problems
}

\author{
Jinxiu Mao $\mathbb{D}^{1},{ }^{1}$ Zengqin Zhao $\mathbb{D}^{1},{ }^{1}$ and Chenguang Wang $\mathbb{D}^{2}$ \\ ${ }^{1}$ School of Mathematics, Qufu Normal University, Qufu, Shandong, 273165, China \\ ${ }^{2}$ Department of Mathematics, Jining University, Qufu, Shandong, 273155, China
}

Correspondence should be addressed to Jinxiu Mao; maojinxiu1982@163.com

Received 21 February 2019; Accepted 31 May 2019; Published 20 June 2019

Guest Editor: Pedro Garrancho

Copyright (c) 2019 Jinxiu Mao et al. This is an open access article distributed under the Creative Commons Attribution License, which permits unrestricted use, distribution, and reproduction in any medium, provided the original work is properly cited.

In this paper, we investigate singular Hadamard fractional boundary value problems. The existence and uniqueness of the exact iterative solution are established only by using an iterative algorithm. The iterative sequences have been proved to converge uniformly to the exact solution, and estimation of the approximation error and the convergence rate have also been derived.

This paper is dedicated to our advisors

\section{Introduction}

Fractional differential operators play an important role in describing phenomenons in many fields such as physics, chemistry, control, and electromagnetism [1-9]. They have many applications in fractional differential equations and fractional integral equations. In [10], authors investigate a class of fractional integral equations arising from a symmetric transition model

$$
\begin{aligned}
& \frac{d}{d t}\left(\frac{1}{2}{ }_{0} D_{t}^{-\beta}\left(u^{\prime}(t)\right)+\frac{1}{2}{ }_{t} D_{T}^{-\beta}\left(u^{\prime}(t)\right)\right)+\nabla F(t, u(t)) \\
& \quad=0, \quad \text { a.e. } t \in[0, T] \\
& u(0)=u(T)=0
\end{aligned}
$$

where ${ }_{0} D_{t}^{-\beta}$ and ${ }_{t} D_{T}^{-\beta}$ are the left and right RiemannLiouville fractional integrals of order $0 \leq \beta<1$, respectively, and $\nabla F(t, x)$ is the gradient of $F$ at $x$.

The fractional order diffusion equation

$$
\frac{\partial^{\alpha} \phi}{\partial t^{\alpha}}=K \frac{\partial^{\beta} \phi}{\partial|x|^{\beta}}
$$

where $0<\alpha \leq 1$ and $1<\beta \leq 2$, contains fractional differential operators which can describe different diffusion process.

In the past ten years, fractional differential equations have been considered in many papers (see [11-22]). Most of the works on the topic have been based on Riemann-Liouville type and Caputo type fractional differential equations. By means of fixed point theorems and variational methods, authors obtain at least one or multiple positive solutions for boundary value problems of fractional differential equations. Very recently, more studies have been carried out on the boundary value problems of nonlinear Hadamard fractional differential equations. An important characteristic of Hadamard fractional derivative is that it contains logarithmic function of arbitrary exponent. However, there are few results about this topic (see [23-28]).

By using the Krasnoselskii-Zabreiko fixed point theorem, Yang [26] obtained at least one positive solution for the boundary value problem

$$
\begin{aligned}
\mathrm{D}^{q} u(t)+f(t, u(t)) & =0, \quad t \in(1, e), \\
u^{(m)}(1) & =0
\end{aligned}
$$




$$
u(e)=\int_{1}^{e} g(t) u(t) \frac{d t}{t}
$$

where $f \in C\left([1, e] \times R^{+}, R\right), g \in C\left([1, e], R^{+}\right)$. $\mathrm{D}^{q}$ was the Hadamard fractional derivative of order $q$. $0 \leq m \leq n-$ 2, $n \in N, n \geq 3, n-1<q \leq n$.

In [28], the authors studied the Hadamard fractional differential equation

$$
\begin{aligned}
{ }^{H} \mathrm{D}^{q} x(t)+\sigma(t) f(t, x(t))= & 0, \\
& 2<q \leq 3, t \in(1,+\infty),
\end{aligned}
$$

with boundary conditions

$$
\begin{gathered}
x(1)=x^{\prime}(1)=0, \\
{ }^{H} \mathrm{D}^{q-1} x(\infty)=a^{H} \mathrm{I}^{\beta} x(\xi)+b \sum_{i=1}^{m-2} \alpha_{i} x\left(\eta_{i}\right), \\
1<\xi<\eta_{1}<\eta_{2}<\cdots<\eta_{m-2}<+\infty
\end{gathered}
$$

where ${ }^{H} \mathrm{D}^{q}$ denoted Hadamard fractional derivative of order $q$ and ${ }^{H} \mathrm{I}^{\beta}$ denoted Hadamard fractional integral of order $\beta . f \in C([1, \infty) \times[0, \infty),[0, \infty)), \sigma:[1, \infty) \longrightarrow[0, \infty)$. By employing the complete continuity of the associated integral operator $T$ and the monotone iterative method, the authors obtained twin positive solutions and the unique positive solution.

Most of the above works required the associated integral operators to be completely continuous because fixed point theorems could be applied. Furthermore, the uniqueness of positive solutions was rarely investigated while the existence and multiplicity of positive solutions were investigated widely.

Inspired by the above results, in this work, we study the existence and uniqueness of positive solutions for the following boundary value problem:

$$
\begin{aligned}
{ }^{H} \mathrm{D}^{q} x(t)+f(t, x(t)) & =0, \quad 2<q \leq 3, t \in(1, e), \\
x(1) & =x^{\prime}(1)=0, \\
x(e) & =0,
\end{aligned}
$$

where ${ }^{H} \mathrm{D}^{q}$ denotes Hadamard fractional derivative of order $q ; f:(1, e) \times[0, \infty) \longrightarrow[0, \infty)$ is continuous.

In this work, only by using the monotone iterative technique, we aim to establish the unique positive solution for problem (6). The main contributions of this work are as follows: (a) the nonlinear term $f(t, x)$ can be singular at $t=1$ and $t=e$; (b) we do not need the continuity and complete continuity of the associated integral operator; (c) we get the unique positive solution.

Throughout this work, we assume that the following conditions hold without further mention.

$\left(H_{1}\right): f:(1, e) \times[0, \infty) \longrightarrow[0, \infty)$ is continuous.
$\left(H_{2}\right)$ : For $(t, x) \in(1, e) \times[0, \infty), f(t, x)$ is nondecreasing in $x$ and there exists a constant $k \in(0,1)$ such that, for $\forall \sigma \in$ $(0,1]$,

$$
f(t, \sigma x) \geq \sigma^{k} f(t, x) .
$$

It is easy to verify that if $\sigma \in(1,+\infty)$, then $f(t, \sigma x) \leq$ $\sigma^{k} f(t, x)$.

\section{Preliminaries}

The way to attack this new problem follows a scheme similar to that used in [21], with the necessary adaptations that Hadamard fractional derivative contains logarithmic function of arbitrary exponent.

In this section, we present some basic concepts and conclusions needed in the proof of our main results.

Definition 1. The Hadamard fractional integral of order $\beta>0$ of a function $x:(1, \infty) \longrightarrow R$ is given by

$$
{ }^{H} \mathrm{I}^{\beta} x(t)=\frac{1}{\Gamma(\beta)} \int_{1}^{t}\left(\log \frac{t}{s}\right)^{\beta-1} \frac{x(s)}{s} d s .
$$

Definition 2. The Hadamard fractional derivative of order $q$ is defined by

$$
\begin{aligned}
& { }^{H} \mathrm{D}^{q} x(t) \\
& =\frac{1}{\Gamma(n-q)}\left(t \frac{d}{d t}\right)^{n} \int_{1}^{t}\left(\log \frac{t}{s}\right)^{n-q-1} \frac{x(s)}{s} d s
\end{aligned}
$$

where $n-1<q \leq n, n=[q]+1, q>0$.

Specifically, ${ }^{H} \mathrm{D}^{n} x(t)=x^{(n)}(t), n=1,2,3, \ldots$.

Lemma 3. Suppose that $h(t) \in C(1, e), 0<\int_{1}^{e} h(s)(d s / s)<$ $+\infty$. Then the Hadamard type fractional differential equation

$$
\begin{aligned}
{ }^{H} \mathrm{D}^{q} x(t)+h(t) & =0, \quad 2<q \leq 3, t \in(1, e), \\
x(1) & =x^{\prime}(1)=0 \\
x(e) & =0
\end{aligned}
$$

has a unique solution

$$
x(t)=\int_{1}^{e} G(t, s) h(s) \frac{d s}{s}
$$

where

$$
\begin{aligned}
& G(t, s)=\frac{1}{\Gamma(q)} \\
& \quad \begin{cases}(\log t)^{q-1}(1-\log s)^{q-1}-\left(\log \left(\frac{t}{s}\right)\right)^{q-1}, & 1 \leq s \leq t \leq e ; \\
(\log t)^{q-1}(1-\log s)^{q-1}, & 1 \leq t \leq s \leq e .\end{cases}
\end{aligned}
$$

Proof. As argued in [9], the solution of the Hadamard differential equation in (10) can be written as the equivalent integral equation

$$
\begin{aligned}
x(t)= & c_{1}(\log t)^{q-1}+c_{2}(\log t)^{q-2}+c_{3}(\log t)^{q-3} \\
& -\frac{1}{\Gamma(q)} \int_{1}^{t}\left(\log \frac{t}{s}\right)^{q-1} h(s) \frac{d s}{s} .
\end{aligned}
$$


From $x(1)=x^{\prime}(1)=0$, we have $c_{3}=c_{2}=0$. Thus (47) reduces to

$$
x(t)=c_{1}(\log t)^{q-1}-\frac{1}{\Gamma(q)} \int_{1}^{t}\left(\log \frac{t}{s}\right)^{q-1} h(s) \frac{d s}{s} .
$$

Using $x(e)=0$ we obtain

$$
c_{1}=\frac{1}{\Gamma(q)} \int_{1}^{e}\left(\log \frac{e}{s}\right)^{q-1} h(s) \frac{d s}{s} .
$$

Substituting (15) into (14), we obtain

$$
\begin{aligned}
x(t)= & (\log t)^{q-1} \cdot \frac{1}{\Gamma(q)} \int_{1}^{e}\left(\log \frac{e}{s}\right)^{q-1} h(s) \frac{d s}{s} \\
& -\frac{1}{\Gamma(q)} \int_{1}^{t}\left(\log \frac{t}{s}\right)^{q-1} h(s) \frac{d s}{s} \\
= & \int_{1}^{e} G(t, s) h(s) \frac{d s}{s} .
\end{aligned}
$$

Take $\rho(t)=(\log t)^{q-1}(1-\log t)$ and $\hat{\rho}(t)=(1-$ $\log t)^{q-1} \log t$ for $q>2, t \in[1, e]$. We can prove that $G(t, s)$ have the following properties.

Lemma 4 (see [26]). For $t, s \in[1, e]$, Green's function $G(t, s)$ satisfies the following properties:

(i) $G(t, s)$ is continuous on $[1, e] \times[1, e]$ and $G(t, s) \geq 0$,

(ii) $\rho(t) \widehat{\rho}(s) \leq \Gamma(q) G(t, s) \leq(q-1) \rho(t)$,

(iii) $\rho(t) \hat{\rho}(s) \leq \Gamma(q) G(t, s) \leq(q-1) \hat{\rho}(s)$,

(iv) $G(t, s)=G(e / t, e / s)$.

From $\Gamma(q+1)=q \Gamma(q), q>0$, and (ii) we get

$$
\rho(t)\left[\frac{1}{\Gamma(q)} \hat{\rho}(s)\right] \leq G(t, s) \leq \frac{1}{\Gamma(q-1)} \rho(t) .
$$

In this paper, we will work in the Banach space $E=C[1, e]$ with the norm $\|x\|=\max _{t \in[1, e]}|x(t)|$.

Define a set $P \subset E$ as follows.

$P=\left\{x \in E \mid\right.$ there are constants $0<l_{x}<1<L_{x}$ such that $\left.l_{x} \rho(t) \leq x(t) \leq L_{x} \rho(t), t \in[1, e]\right\}$. Evidently $\rho(t) \in P$. Therefore, $P$ is not empty.

\section{The Main Results}

Theorem 5. Assume $\left(H_{1}\right),\left(H_{2}\right)$ hold. And

$$
0<\int_{1}^{e} f(t, \rho(t)) \frac{d t}{t}<\infty .
$$

Then problem (6) has at least one positive solution $x^{*}(t)$.

Proof. Define the operator $T: E \longrightarrow E$ by

$$
T x(t)=\int_{1}^{e} G(t, s) f(s, x(s)) \frac{d s}{s} .
$$

We can see easily the equivalence between $x$ is a solution of (6) and $x$ is a fixed point of $T$.
Claim 1. The operator $T: P \longrightarrow P$ is nondecreasing.

In fact, for $x \in P$, it is obvious that $T x \in E, T x(1)=$ $T x(e)=0$. For any $x \in P$ and $t \in[1, e]$, from (17),

$$
\begin{aligned}
T x(t) & =\int_{1}^{e} G(t, s) f(s, x(s)) \frac{d s}{s} . \\
& \leq \int_{1}^{e} \frac{1}{\Gamma(q-1)} \rho(t) f\left(s, L_{x} \rho(s)\right) \frac{d s}{s} \\
& \leq \rho(t) L_{x}^{k} \frac{1}{\Gamma(q-1)} \int_{1}^{e} f(s, \rho(s)) \frac{d s}{s} \\
& \leq L_{T x} \rho(t)
\end{aligned}
$$

and

$$
\begin{aligned}
T x(t) & =\int_{1}^{e} G(t, s) f(s, x(s)) \frac{d s}{s} . \\
& \geq \int_{1}^{e} \frac{1}{\Gamma(q)} \hat{\rho}(s) \rho(t) f\left(s, l_{x} \rho(s)\right) \frac{d s}{s} \\
& \geq \rho(t) l_{x}^{k} \frac{1}{\Gamma(q)} \int_{1}^{e} \hat{\rho}(s) f(s, \rho(s)) \frac{d s}{s} \\
& =l_{T x} \rho(t),
\end{aligned}
$$

where $L_{T x}$ and $l_{T x}$ are positive constants satisfying

$$
\begin{aligned}
L_{T x} & >\max \left\{1, L_{x}^{k} \frac{1}{\Gamma(q-1)} \int_{1}^{e} f(s, \rho(s)) \frac{d s}{s}\right\}, \\
0 & <l_{T x}<\min \left\{1, l_{x}^{k} \frac{1}{\Gamma(q)} \int_{1}^{e} \hat{\rho}(s) f(s, \rho(s)) \frac{d s}{s}\right\} .
\end{aligned}
$$

Thus, it follows that there are constants $0<l_{T x}<1<L_{T x}$ such that, for $t \in[1, e]$,

$$
l_{T x} \rho(t) \leq T x(t) \leq L_{T x} \rho(t) .
$$

Therefore, for any $x \in P, T x \in P, T$ is the operator $P \longrightarrow P$. From (19), it is easy to see that $T$ is nondecreasing with respect to $x$. Hence, Claim 1 holds.

Claim 2. There exist a nondecreasing sequence $\left\{u_{n}\right\}$ and a nonincreasing sequence $\left\{v_{n}\right\}$ and there exists $x^{*} \in P$ such that

$$
\begin{aligned}
& u_{n}(t) \longrightarrow x^{*}(t), \\
& v_{n}(t) \longrightarrow x^{*}(t),
\end{aligned}
$$

uniformly on $[1, e]$.

First, there exist two constants $l_{T \rho}, L_{T \rho}$ with $0<l_{T \rho}<1<$ $L_{T \rho}$ since $T \rho \in P$. Take $\delta$ and $\gamma$ to be fixed numbers satisfying

$$
\begin{aligned}
& 0<\delta \leq l_{T \rho}^{1 /(1-k)}, \\
& \gamma \geq L_{T \rho}^{1 /(1-k)} .
\end{aligned}
$$

Obviously, $0<\delta<1<\gamma$. 
We construct two iterative sequences as follows:

$$
\begin{aligned}
u_{0}(t) & =\delta \rho(t), \\
v_{0}(t) & =\gamma \rho(t), \\
u_{n} & =T u_{n-1}, \\
v_{n} & =T v_{n-1},
\end{aligned}
$$

$$
n=1,2,3, \ldots
$$

Then

$$
u_{0} \leq u_{1} \leq \ldots \leq u_{n} \leq \ldots v_{n} \leq \ldots \leq v_{1} \leq v_{0} .
$$

In fact, from (26), we have $u_{0}, v_{0} \in P$ and $u_{0} \leq v_{0}$. Furthermore,

$$
\begin{aligned}
u_{1}(t) & =T u_{0}(t)=\int_{1}^{e} G(t, s) f(s, \delta \rho(s)) \frac{d s}{s} \\
& \geq \delta^{k} \int_{1}^{e} G(t, s) f(s, \rho(s)) \frac{d s}{s}=\delta^{k} T \rho \\
& \geq \delta^{k} l_{T \rho} \rho(t) \geq \delta^{k} \delta^{1-k} \rho(t)=u_{0}(t), \\
v_{1}(t) & =T v_{0}(t)=\int_{1}^{e} G(t, s) f(s, \gamma \rho(s)) \frac{d s}{s} \\
& \leq \gamma^{k} \int_{1}^{e} G(t, s) f(s, \rho(s)) \frac{d s}{s}=\gamma^{k} T \rho \\
& \leq \gamma^{k} L_{T \rho} \rho(t) \leq \gamma^{k} \gamma^{1-k} \rho(t)=v_{0}(t) .
\end{aligned}
$$

From $u_{0} \leq v_{0}$ and $T$ nondecreasing, (28) holds. Let $c_{0}=\delta / \gamma$; then $0<c_{0}<1$. It follows from (7) that

$$
T\left(c_{0} u\right) \geq c_{0}^{k} T u
$$

And, for any natural number $n$,

$$
\begin{aligned}
u_{n} & =T u_{n-1}=T^{n} u_{0}=T^{n}(\delta \rho)=T^{n}\left(c_{0} \gamma \rho\right) \\
& \geq c_{0}^{k^{n}} T^{n}(\gamma \rho)=c_{0}^{k^{n}} v_{n} .
\end{aligned}
$$

Thus, for any natural number $n$ and $p^{*}$, we have

$$
\begin{aligned}
0 & \leq u_{n+p^{*}}-u_{n} \leq v_{n}-u_{n} \leq\left(1-c_{0}^{k^{n}}\right) v_{n} \\
& \leq\left(1-c_{0}^{k^{n}}\right) \gamma \rho,
\end{aligned}
$$

which implies that $\left\{u_{n}\right\}$ is a cauchy sequence in $\left[u_{0}, v_{0}\right]$. So there exists $x^{*} \in\left[u_{0}, v_{0}\right] \subset P$ such that

$$
u_{n}(t) \longrightarrow x^{*}(t)
$$

and from (32)

$$
v_{n}(t) \longrightarrow x^{*}(t) .
$$

From $x^{*} \in\left[u_{0}, v_{0}\right]$, we have $T x^{*} \in\left[u_{0}, v_{0}\right]$. Combining with $T$ nondecreasing on $x$,

$$
u_{n} \leq T x^{*} \leq v_{n}
$$

Let $n \longrightarrow \infty$,

$$
x^{*}(t)=T x^{*}(t),
$$

which implies $x^{*}$ is a positive solution of problem (6).

Theorem 6. Assume $\left(H_{1}\right),\left(H_{2}\right)$ hold. Then, we have the following:

(i)Problem (6) has unique positive solution $x^{*}(t)$ in $P$ and there exist constants $l, L$ with $0<l<1<L$ such that

$$
l \rho(t) \leq x^{*}(t) \leq L \rho(t), \quad t \in[1, e] .
$$

(ii)For any initial value $x_{0}(t) \in P$, there exists a sequence $\left\{x_{n}(t)\right\}$ that uniformly converges to the unique positive solution $x^{*}(t)$, and we have the error estimation

$$
\max \left|x_{n}(t)-x^{*}(t)\right|=o\left(1-\lambda^{k^{n}}\right),
$$

where $\lambda$ is a constant with $0<\lambda<1$ and determined by $x_{0}$; $o\left(1-\lambda^{k^{n}}\right)$ represents the same order infinitesimal of $\left(1-\lambda^{k^{n}}\right)$.

Proof. Let $u_{0}, v_{0}, u_{n}, v_{n}$ be defined in (26) and (27).

(i) It follows from Theorem 5 that problem (6) has a positive solution $x^{*}(t) \in P$, which implies that there exists constants $l$ and $L$ with $0<l<L<1$ such that

$$
l \rho(t) \leq x^{*}(t) \leq L \rho(t), \quad t \in[1, e] .
$$

Let $\bar{x}(t) \in P$ be another positive solution of problem (6). Then there exist constants $c_{1}$ and $c_{2}$ with $0<c_{1}<1<c_{2}$ such that

$$
c_{1} \rho(t) \leq \bar{x}(t) \leq c_{2} \rho(t), \quad t \in[1, e] .
$$

Let $\delta$ defined in (25) be small enough so that $\delta<c_{1}$ and $\gamma$ defined in (25) be large enough so that $\gamma>c_{2}$. Then

$$
u_{0}(t) \leq \bar{x}(t) \leq v_{0}(t), \quad t \in[1, e] .
$$

Note that $T \bar{x}(t)=\bar{x}(t)$ and $T$ is nondecreasing; we have

$$
u_{n}(t) \leq \bar{x}(t) \leq v_{n}(t), \quad t \in[1, e] .
$$

Letting $n \longrightarrow \infty$, we obtain that $x^{*}(t)=\bar{x}(t)$. Hence, the positive solution of problem (6) is unique.

(ii) For any $x_{0}(t) \in P$, there exist constants $l_{0}$ and $L_{0}$ with $0<l_{0}<1<L_{0}$ such that

$$
l_{0} \rho(t) \leq x_{0}(t) \leq L_{0} \rho(t), \quad t \in[1, e] .
$$

Similar to (i), take $\delta$ and $\gamma$ to be defined by (25) satisfying $\delta<l_{0}$ and $\gamma>L_{0}$. Then

$$
u_{0}(t) \leq x_{0}(t) \leq v_{0}(t), \quad t \in[1, e] .
$$

Let

$$
\begin{aligned}
x_{n}(t)=T x_{n-1}(t)=\int_{1}^{e} G(t, s) f\left(s, x_{n-1}(s)\right) \frac{d s}{s}, & \\
n & =1,2, \ldots .
\end{aligned}
$$

Note that $T$ is nondecreasing,

$$
u_{n}(t) \leq x_{n}(t) \leq v_{n}(t), \quad t \in[1, e] .
$$

Letting $n \longrightarrow \infty$, it follows from (33) and (34) that $x_{n}(t) \longrightarrow$ $x^{*}(t)$ uniformly on $[1, e]$.

At the same time, (38) follows from (32). 
Remark 7. We just investigate a simple form of boundary value problems for Hadamard differential equations. We can easily apply the monotone iterative technique to multipoint or multistrip boundary value problems.

Remark 8. Suppose that $\beta_{i}(t)(i=0,1,2, \ldots m)$ are nonnegative continuous functions on $(1, e)$, which may be unbounded at the end points of $(1, e) . \Omega$ is the set of functions $f(t, x)$ which satisfy the conditions $\left(H_{1}\right)$ and $\left(H_{2}\right)$. Then we have the following conclusions:

(1) $\beta_{i}(t) \in \Omega, x^{b} \in \Omega$, where $0<b<1$.

(2) If $0<b_{i}<+\infty(i=1,2, \ldots m)$ and $b>\max _{1 \leq i \leq m}\left\{b_{i}\right\}$, then $\left[\beta_{0}(t)+\sum_{i=1}^{m} \beta_{i}(t) x^{b_{i}}\right]^{1 / b} \in \Omega$.

(3) If $f(t, x) \in \Omega$, then $\beta_{i}(t) f(t, x) \in \Omega$.

(4) If $f_{i}(t, x) \in \Omega(i=1,2, \ldots m)$, then $\max _{1 \leq i \leq m}\left\{f_{i}(t, x)\right\}$ $\in \Omega, \min _{1 \leq i \leq m}\left\{f_{i}(t, x)\right\} \in \Omega$.

The above four facts can be verified directly. This indicates that there are many kinds of functions which satisfy the conditions $\left(H_{1}\right)$ and $\left(H_{2}\right)$.

\section{An Example}

Consider the following boundary value problem:

$$
\begin{aligned}
{ }^{H} \mathrm{D}^{5 / 2} u(t)+a(t) u^{1 / 4}+b(t) u^{1 / 3} & =0, \quad t \in(1, e), \\
u(1) & =u^{\prime}(1)=0, \\
u(e) & =0,
\end{aligned}
$$

where $q=5 / 2, f(t, u)=a(t) u^{1 / 4}+b(t) u^{1 / 3}, a(t), b(t) \in C((1$, $e),(0,+\infty))$.

Analysis 1. First, $f \in C((1, e) \times[0, \infty),[0, \infty))$ and so $\left(H_{1}\right)$ holds.

For any $\sigma \in(0,1)$, we take $k=1 / 2$ and have

$$
f(t, \sigma u) \geq \sigma^{k} f(t, u) .
$$

Then $\left(H_{2}\right)$ holds.

Obviously

$$
0<\int_{1}^{e} f(t, \rho(t)) \frac{d t}{t}<\infty
$$

where $\rho(t)=(\log t)^{5 / 2-1}(1-\log t)$. Hence all conditions of Theorem 5 are satisfied, and consequently we have the following corollary.

Corollary 9. Problem (47) has unique positive solution $u^{*}(t)$. For any initial value $x_{0} \in P$, the successive iterative sequence $\left\{x_{n}(t)\right\}$ generated by

$$
\begin{aligned}
x_{n}(t)=\int_{0}^{1} G(t, s)\left(a(s) u^{1 / 4}+b(s) u^{1 / 3}\right) \frac{d s}{s} & \\
n & =1,2, \ldots
\end{aligned}
$$

uniformly converges to the unique positive solution $u^{*}(t)$ on $[1, e]$. We have the error estimation

$$
\max \left|x_{n}(t)-u^{*}(t)\right|=\circ\left(1-\lambda^{(1 / 2)^{n}}\right),
$$

where $\lambda$ is a constant with $0<\lambda<1$ and determined by the initial value $x_{0}$. And there are constants $l, L$ with $0<l<1<L$ such that

$$
l \rho(t) \leq u^{*}(t) \leq L \rho(t), \quad t \in[1, e] .
$$

\section{Data Availability}

Data sharing is not applicable to this article as no datasets were generated or analysed during the current study.

\section{Conflicts of Interest}

The authors declare that they have no conflicts of interest.

\section{Acknowledgments}

This paper is supported by the Natural Science Foundation of China (11571197) and the Science Foundation of Qufu Normal University of China (XJ201112).

\section{References}

[1] K. Diethelm and A. Freed, "On the solution of nonlinear fractional order differential equations used in the modeling of viscoelasticity," in Scientific Computing in Chemical Engineering II-Computational Fluid Dynamics, Reaction Engineering and Molecular Properties, F. Keil, W. Mackens, H. Voss, and J. Werther, Eds., pp. 217-224, Springer-Verlag, Heidelberg, Germany, 1999.

[2] B. N. Lundstrom, M. H. Higgs, W. J. Spain, and A. L. Fairhall, "Fractional differentiation by neocortical pyramidal neurons," Nature Neuroscience, vol. 11, no. 11, pp. 1335-1342, 2008.

[3] W. G. Glockle and T. F. Nonnenmacher, "A fractional calculus approach to self-similar protein dynamics," Biophysical Journal, vol. 68, no. 1, pp. 46-53, 1995.

[4] R. Hilfer, Applications of Fractional Calculus in Physics, World Scientific, Singapore, 2000.

[5] A. Arafa, S. Rida, and M. Khalil, "Fractional modeling dynamics of HIV and CD4+ T-cells during primary infection," Nonlinear Biomedical Physics, vol. 6, no. 1, pp. 1-7, 2012.

[6] J. W. Kirchner, X. Feng, and C. Neal, "Frail chemistry and its implications for contaminant transport in catchments," Nature, vol. 403, no. 6769, pp. 524-527, 2000.

[7] D. A. Benson, S. W. Wheatcraft, and M. M. Meerschaert, "Application of a fractional advection-dispersion equation," Water Resources Research, vol. 36, no. 6, pp. 1403-1412, 2000.

[8] D. A. Benson, S. W. Wheatcraft, and M. M. Meerschaert, "The fractional-order governing equation of Lévy motion," Water Resources Research, vol. 36, no. 6, pp. 1413-1423, 2000.

[9] I. Podlubny, Fractional Differential Equations, vol. 198 of Mathematics in Science and Engineering, Academic Press, New York, NY, USA, 1999.

[10] X. Zhang, L. Liu, and Y. Wu, "Variational structure and multiple solutions for a fractional advection-dispersion equation," Computers \& Mathematics with Applications, vol. 68, no. 12, part A, pp. 1794-1805, 2014.

[11] Y. Wang and L. Liu, "Positive solutions for a class of fractional 3 -point boundary value problems at resonance," Advances in Difference Equations, vol. 2017, no. 13, 2017. 
[12] Z. Yue and Y. Zou, "New uniqueness results for fractional differential equation with dependence on the first order derivative," Advances in Difference Equations, vol. 2019, no. 1, p. 38, 2019.

[13] K. M. Zhang, "On a sign-changing solution for some fractional differential equations," Boundary Value Problems, vol. 2017, no. 59, 2017.

[14] Y. Guan, Z. Zhao, and X. Lin, "On the existence of positive solutions and negative solutions of singular fractional differential equations via global bifurcation techniques," Boundary Value Problems, vol. 2016, no. 141, pp. 1-18, 2016.

[15] Y. L. Guan, Z. Q. Zhao, and X. Lin, "On the existence of solutions for impulsive fractional differential equations," Advances in Mathematical Physics, vol. 2017, Article ID 1207456, 12 pages, 2017.

[16] J. Jiang, W. Liu, and H. Wang, "Positive solutions for higher order nonlocal fractional differential equation with integral boundary conditions," Journal of Function Spaces, vol. 2018, Article ID 6598351, 12 pages, 2018.

[17] J. Q. Jiang, W. W. Liu, and H. C. Wang, "Positive solutions to singular dirichlet-type boundary value problems of nonlinear fractional differential equations," Advances in Difference Equations, vol. 2018, no. 169, 2018.

[18] X. S. Du and A. M. Mao, "Existence and multiplicity of nontrivial solutions for a class of semilinear fractional Schrödinger equations," Journal of Function Spaces, vol. 2017, Article ID 3793872, 7 pages, 2017.

[19] J. X. Mao and Z. Q. Zhao, "The existence and uniqueness of positive solutions for integral boundary value problems," Bulletin of the Malaysian Mathematical Sciences Society, vol. 34, no. 1, pp. 153-164, 2011.

[20] J. X. Mao and Z. Q. Zhao, "On existence and uniqueness of positive solutions for integral boundary boundary value problems," Electronic Journal of Qualitative Theory of Differential Equations, vol. 16, pp. 1-8, 2010.

[21] J. X. Mao, Z. Q. Zhao, and C. G. Wang, "The exact iterative solution of fractional differential equation with nonlocal boundary value conditions," Journal of Function Spaces, vol. 2018, Article ID 8346398, 6 pages, 2018.

[22] J. X. Mao, Z. Q. Zhao, and A. X. Qian, "Laplace's equation with concave and convex boundary nonlinearities on an exterior region," Boundary Value Problems, vol. 51, pp. 1-12, 2019.

[23] K. Y. Zhang and Z. Q. Fu, "Solutions for a class of Hadamard fractional boundary value problems with sign-changing nonlinearity," Journal of Function Spaces, vol. 2019, Article ID 9046472 , 7 pages, 2019.

[24] J. Tariboon, S. K. Ntouyas, S. Asawasamrit, and C. Promsakon, "Positive solutions for Hadamard differential systems with fractional integral conditions on an unbounded domain," Open Mathematics, vol. 15, no. 1, pp. 645-666, 2017.

[25] B. Ahmad and S. Ntouyas, "A fully Hadamard type integral boundary value problem of a coupled system of fractional differential equations," Fractional Calculus and Applied Analysis, vol. 17, no. 2, pp. 348-360, 2014.

[26] W. G. Yang and Y. P. Qin, "Positive solutions for nonlinear Hadamard fractional differential equations with integral boundary conditions," ScienceAsia, vol. 43, no. 3, pp. 201-206, 2017.

[27] Q. Ma, R. Wang, J. Wang, and Y. Ma, "Qualitative analysis for solutions of a certain more generalized two-dimensional fractional differential system with Hadamard derivative," Applied Mathematics and Computation, vol. 257, pp. 436-445, 2015.
[28] G. Wang, K. Pei, R. P. Agarwal, L. Zhang, and B. Ahmad, "Nonlocal hadamard fractional boundary value problem with hadamard integral and discrete boundary conditions on a halfline," Journal of Computational and Applied Mathematics, vol. 343, pp. 230-239, 2018. 


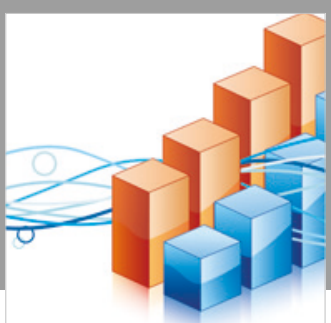

Advances in

Operations Research

\section{-n-m}
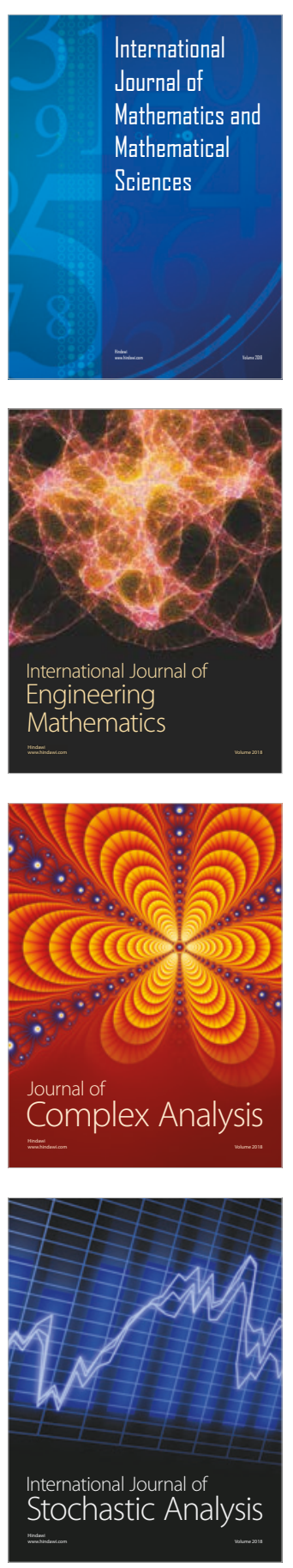
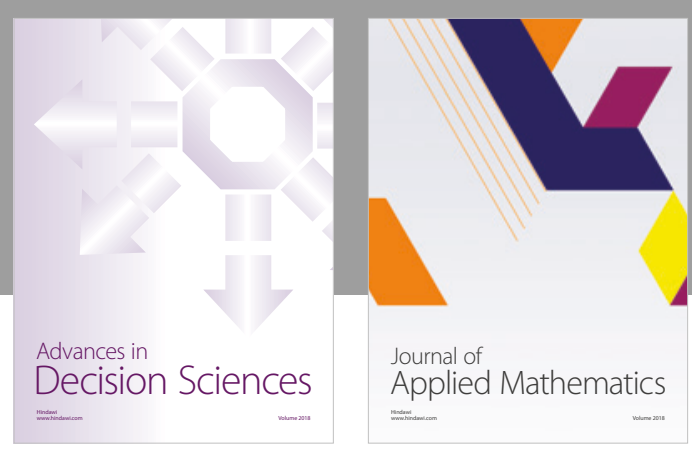

Journal of

Applied Mathematics
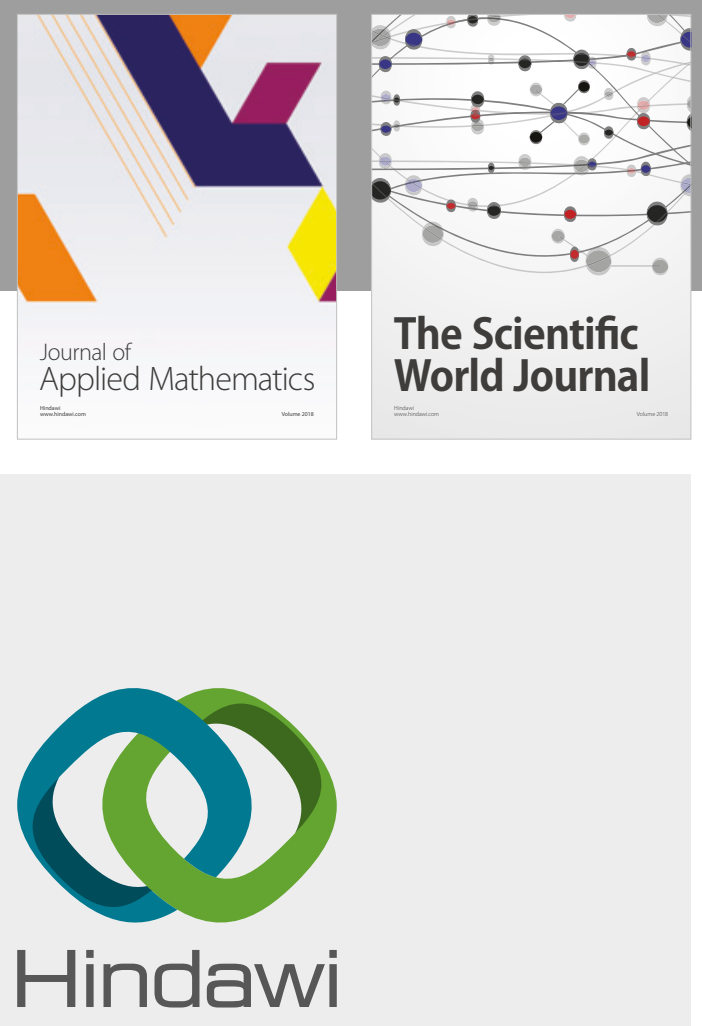

Submit your manuscripts at

www.hindawi.com

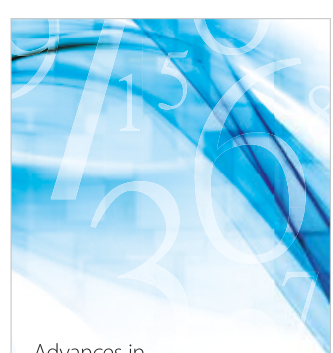

Advances in
Numerical Analysis
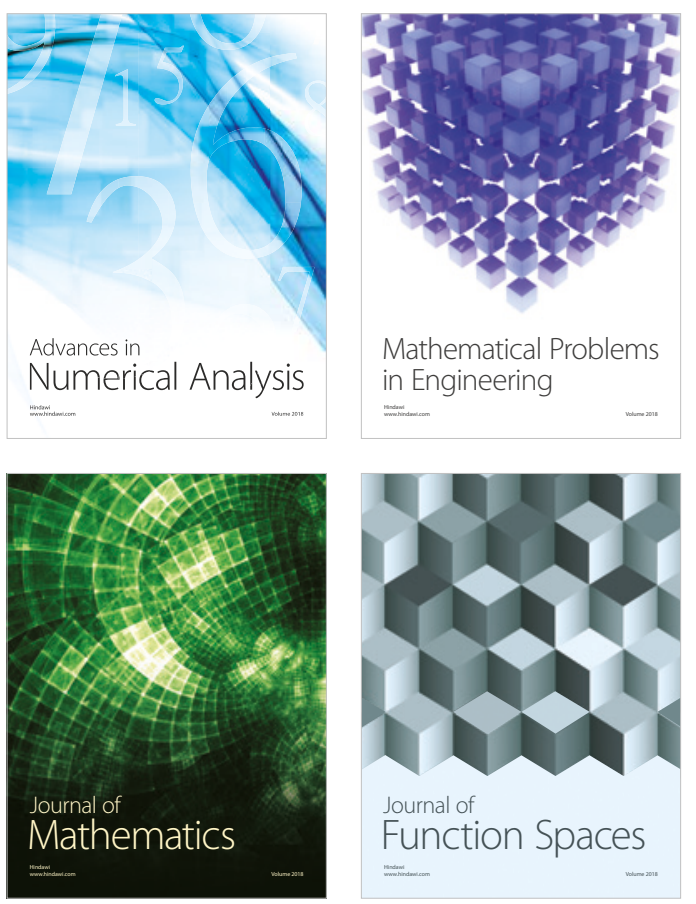

Mathematical Problems in Engineering

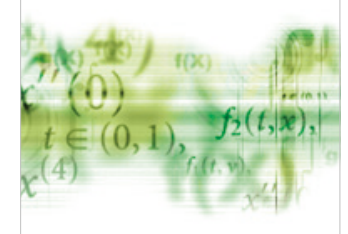

International Journal of

Differential Equations

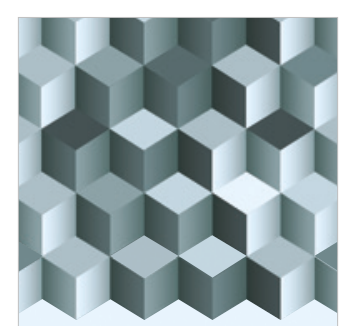

Journal of

Function Spaces

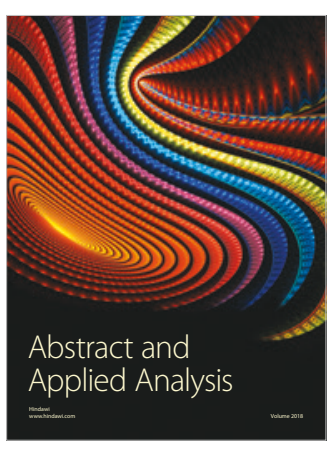

The Scientific

World Journal

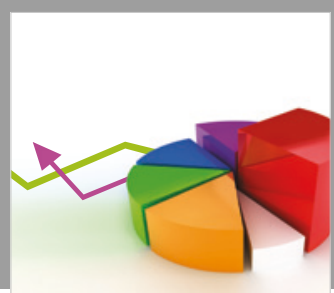

Journal of

Probability and Statistics
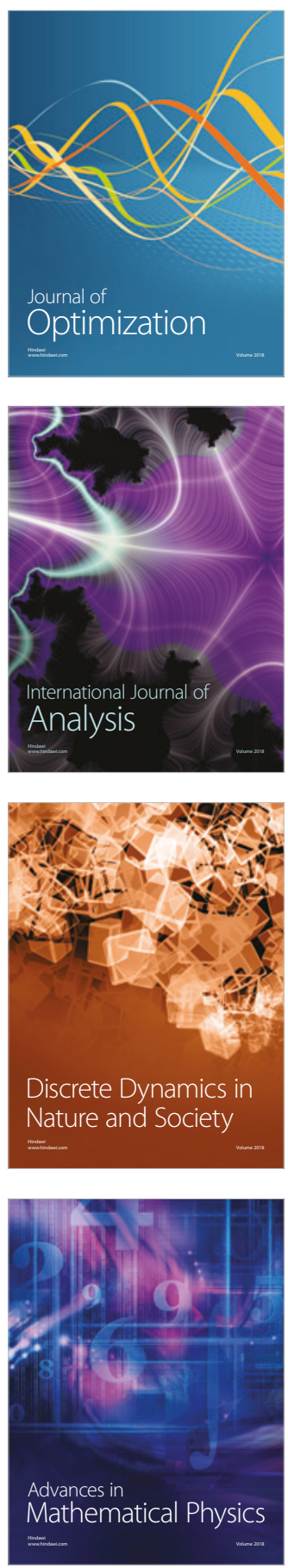